\title{
Domain Motion in 3-isopropylmalate dehydrogenase: A Strategy to Enhance its Thermal Stability
}

\author{
Zeily Nurachman*, Tairo Oshima ${ }^{\dagger} \&$ Nobuo Tanaka \\ *Department of Chemistry, Institut Teknologi Bandung, \\ Jl. Ganesha 10, Bandung 40132, Indonesia \\ 'Department of Molecular Biology, Tokyo University of Pharmacy and Life Science, \\ Horinouchi, Hachioji 192-0392, Japan \\ ${ }^{\ddagger}$ Graduate School of Bioscience and Biotechnology, Tokyo Institute of Technology, \\ Nagatsuta 4259, Yokohama 226-8501, Japan
}

\begin{abstract}
In order to elucidate the thermal properties of Thermus thermophilus 3 -isopropylmalate dehydrogenase, mutant structures with mutations at the Cterminus were compared with each other. The structural movement can be anticipated from the structural changes among mutants in regions of a minor groove and pillar. Our previous studies revealed that the open-close movement of the active site groove antagonizes to that of the minor groove (like a paperclip) and the thermostability of the enzyme increases when the active site groove is closed. In the present study, it is shown that the motion of the enzyme mainly occurs in the first domain and strand $\mathrm{D}$ in the pillar structure is a hingebending region of the movement. The motion of the first domain to expand the minor groove may close the active site groove suggesting a mechanism for the enhanced thermal stability of 3-isopropylmalate dehydrogenase.
\end{abstract}

Keywords: domain movement; enzyme mechanisms; induced enzyme; paperclip-like motion; protein design; thermal stability.

\section{Introduction}

3-Isopropylmalate dehydrogenase (IPMDH, EC 1.1.1.85), a product of the leuB gene, catalyzes dehydration at $\mathrm{C} 2$ and decarboxylation at $\mathrm{C} 3$ of $(2 R, 3 S)-3-$ isopropylmalate (IPM) to 2-oxoisocaproate in the third step of the leucinebiosynthesis pathway in microorganisms and plants. The genes of the enzyme have been cloned and sequenced from various organisms with a variety of growth temperatures including psychrotrophic (cold-loving), mesotrophic, and thermotrophic organisms (1-8).

The structure of thermophilic IPMDH from T. thermophilus has been reported (9) and has been compared with their mesophilic counterparts (10-12). The thermostable IPMDH is identified with intrinsic properties, e.g. high proline content, low solvent-exposed surface area, high ion pairs and extra salt bridges. T. thermophilus IPMDH is a homodimer (subunits A and B hereafter) and each 
monomer is composed of two domains: first domain and second domain. The active site and minor grooves of the enzyme are constituted between domains and are located to each other in the place of the contrary.

We previously reported that the minor groove contributes to the thermal stability (13). We demonstrated possible mechanisms for partial restoration of thermostability in IPMDH mutants at the C-terminus. A mutant having a wide minor groove is able to recover the heat resistant; the enzyme is more thermostable when the minor groove is more expanded. We also defined three kinds of intramolecular interactions, that is, pillar structures, dimer interface structures, and covering-pillar structures. In the present study, we further explore structural changes among the thermostable IPMDH mutants particularly in regions of a minor groove and pillar that play roles on the plausible movements of the enzyme structure. The enzymes presented in this study are shown in Figure 1, have different thermostabilities without any drastically changes in the functional properties. Based on the structural variations of minor groove and pillar in these mutant enzymes, we propose a mechanism for increasing in the enzyme thermostability.

$\begin{array}{llll}\text { HD177 } & 341 & \text { GMGI } 344 \\ \text { HD708 } & 341 \text { TATVGI 346 } \\ \text { HD711 } & 341 \text { EAFTATVGI } 349 \\ \text { A172L } & 341 \text { LRHLA 345 }\end{array}$

Figure 1 Amino acid sequence alignment of T. thermophilus IPMDHs: A172L, HD711, HD708, and HD177 in the carboxy-terminal region.

\section{Materials and Methods}

Three structures of modified IPMDHs at the C-terminus have been solved and their atomic coordinates and structure factors have been deposited at the Protein Data Bank (PDB, http://www.rcsb.org) with the accession code 1DPZ, 1DR0, and 1DR8 for HD711, HD708, and HD177, respectively (13). The coordinates of the original-type of enzyme, A172L, have also been deposited previously at the PDB with the code 1OSJ (14). The amino acid sequences of all enzymes differ only in the C-terminal regions (Figure 1) and their thermal stabilities reduce in an order of A172L $>$ HD711 $>$ HD708 $>$ HD177 (15). Structures were viewed and oriented using the program TURBO-FRODO (16) on an Indigo2 computer (Silicon Graphics). The structures are evaluated using the program QUANTA (Molecular Simulations Inc.) or the program XPLOR (17) and redrawn using the program MOLSCRIPT (18). The regions examined included the residues shown in Table 1. 


\begin{tabular}{lll}
\hline Regions & Structures & Residues \\
\hline Minor groove & Helix e & K159, P160, E161, V162, E163, R164, V165, A166, \\
(domain 2 part) & & R167, V168, A169, F170, E171, L172, A173, R174 \\
Minor groove & Helix j & V305, E306, L307, A308, A309, K310, V311, E312, \\
(domain 1 part) & & D313, A314, V315, A316, K317, A318, L319, L320, \\
& & E321 \\
Pillar & Strand D & P267, V268, F269, E270 \\
\hline
\end{tabular}

Table 1 Residues in regions of minor groove and pillar.

\section{$3 \quad$ Results and Discussion}

Overall Structure View Each structure of four IPMDHs is composed of two identical subunits, each of which has a similar folding topology with each other (Figure 2). Each monomer consists of two domains: first domain has five $\beta$ strands (A, B, C, D, and E) and seven $\alpha$-helices (a, b, c, d, i, j, and k), and second domain has seven $\beta$-strands (F, G, H, I, K, and L) and four $\alpha$-helices (e, $\mathrm{f}, \mathrm{g}$, and $\mathrm{h})$, respectively. The structure is redrawn in a schematic diagram for clarity (Figure 3 ).

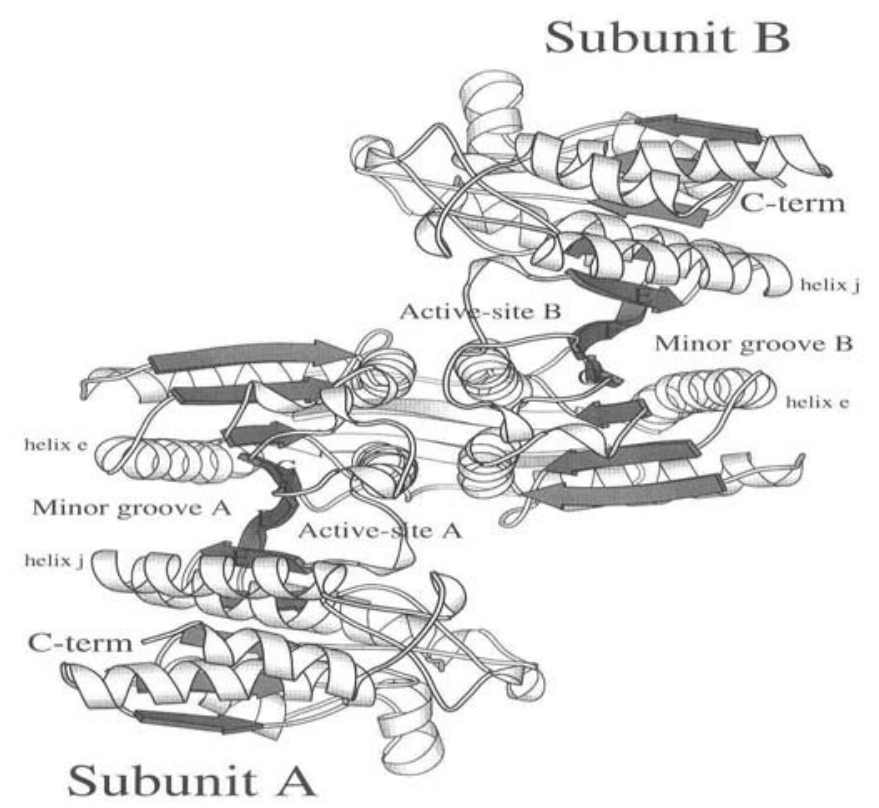

Figure 2 Ribbon representation of overall structure of dimer IPMDH. Shaded arrows denote IPMDH pillars. 


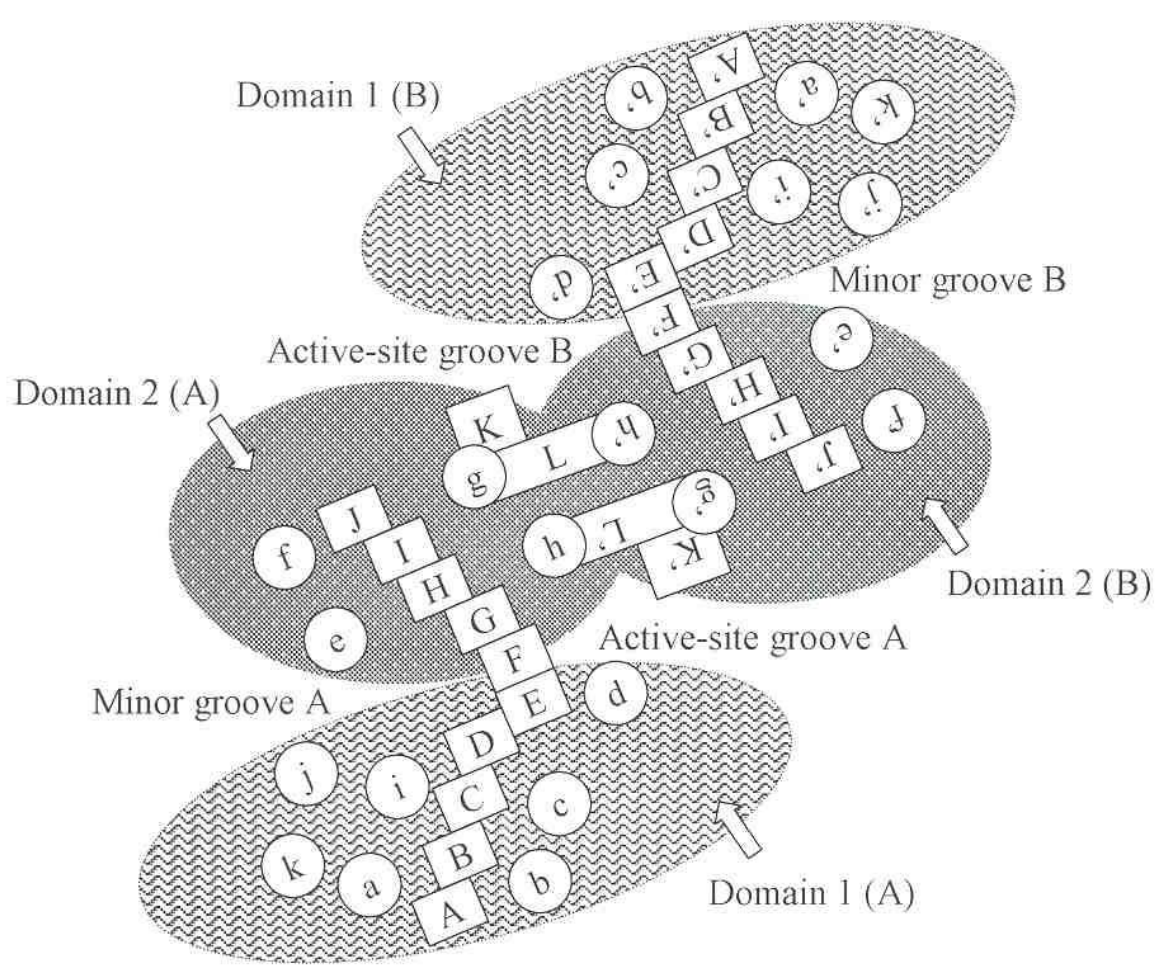

Figure 3 Schematic diagram of dimer IPMDH. Circles (lower-case letters) and boxes (upper-case letters) represent $\alpha$-helices and $\beta$-strands, respectively.

For the analysis of thermostability, the monomer is imagined as divided into three parts, that is, pillar structure (strands A to J), dimer interface structure (strand L, helices g and h, and loop FG), and covering-pillar structure (helices and loops surrounding the pillar). The pillar structure is rigid and far from the solvent. An active site groove is buried between the first domain and the dimer interface (Figure 2 and 3). The opposite side of the active site groove is a minor groove. The open and close movements of both grooves like a paperclip motion through the pillar, by which the minor groove is compressed when the active site groove is expanded or vice versa. The regions analyses of IPMDH were used to choose structures involved in the motion. Three region groups were identified from the regional analyses of IPMDH (Table 1) and the comparison of alteration in these regions revealed an "open-close" mechanism of the grooves in IPMDH. 
Arrangement Residues in the Minor Groove There are no drastic changes in catalytic activity and major intramolecular interactions among A172L, HD711, HD708, and HD177. The changes of thermostability in the mutants are, as a result of domain movements, that it can be measured from the change of the width of minor or active site groove. Minor groove is a space covered by two helices (e and j) exposed to the solvent and three strands (D, E, and F) hidden inside the subunit (Figure 2 and 4). Figure 4 also depicts the directions of minor groove horizontally. No residue differences exist among mutants A172L, HD711, HD708, and HD177 except for the width of minor groove. The widths of the groove in subunit A differ from that in subunit B and are adjusted through the helices e and $\mathrm{j}$ (the solvent-exposed helices on the minor groove).

Helix e obstructs the pillar (strands F, G, H, I, and J) from the solvent to be shifted toward helix f, not into the mouth of minor groove (Figure 2 and 3), and had no significant differences among mutants A172L, HD711, HD708, and HD177 in its accessible surface. It was found that the helix e indirectly contributed to dimer-interface interactions. Residue of K159 at the bottom of helix e attaches to the end of strand L (long arm-like strand for dimer interactions). This residue makes salt bridges with residues of E201 (helix f) and E163 and seems to lock the end of strand L position. The successions of ionic residues of K159, E163, R167, E171, and R174 cover the exterior of helix e facing to the solvent. These residues also stabilize the helix with the electrostatic networks in each turn of helix. Residues of E161 and R164 also stabilize the helix turn in the bottom region and salt bridges among R164, E98 (loop d-F), and R264 (loop E-D) seem to wrap the arrangement of strands D, E, and $\mathrm{F}$. Residue of P160 prevents the placement of helix e in the minor groove because of it strict conformation.

The interior of helix e facing to the pillar consists of hydrophobic residues of V162, V165, A166, V168, A169, F170, L172, and A173. The small size of side chain of these residues except F170 is indispensable to maintain the helix structure. Beside that, the hydrophobic properties are necessary to interact with the pillar. Residue of F170 (close to the top of helix e) seems to have a unique position to fill up the hydrophobic pocket of V180 (strand I), V199, V202 (helix f), V209, and L211 (strand J).

To maintain the pillar in the region of second domain of IPMDH, helix e must be conserved in the enzyme structure. Mutations at position 172 of IPMDH (e.g. A172L, A172E, A172N, and A172Q) show exciting results that it may explain the importance for maintaining helix e in the structure $(14,19)$. Residue of L172 in A172L-mutant elongates a hydrophobic network of V165 and V168 of the interior helix e in each turn and also interacts with residues V232 (strand F) and L129 (strand G) resulting an inflexible second domain to increase its thermal 
stability (14). In the case of HD711, HD708, and HD177, the mutations in the $\mathrm{C}$-terminal region cause the compressed minor groove, which suggests why the thermostability is decreased (13).

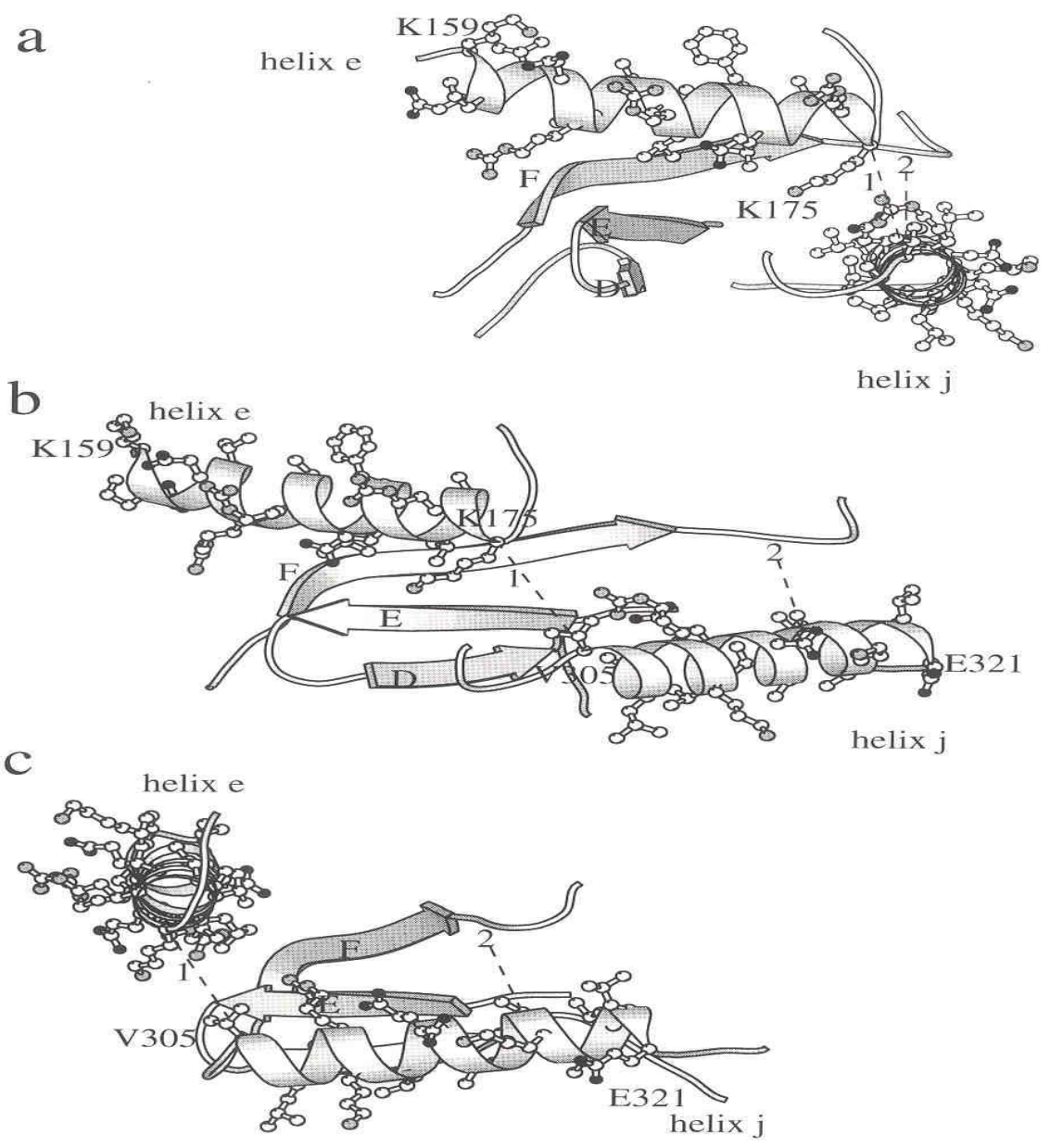

Figure 4 Residual arrangement of helices $e$ and $\mathrm{j}$ in the minor groove of IPMDH. White, shaded, and black balls denote atoms of $\mathrm{C}, \mathrm{N}$, and $\mathrm{O}$, respectively. A rotated figure (a) about $45^{\circ}$ and $90^{\circ}$ horizontally results figures (b) and (c), respectively. (1) and (2) represent the width of minor groove.

Although helix $\mathrm{j}$ is not involved in the masking pillar structure directly (Figure 2 and 3), it is located at the attractive position parallel to the strand D (a part of pillar structure in the first domain). A closest distance to helix $\mathrm{j}$ in the minor 
groove is subject to measure the width of the groove. The intensive contacts among helices $\mathrm{j}, \mathrm{i}$, a, and $\mathrm{k}$ make inflexibility in the first domain of IPMDH. In the top region of helix $j$, salt bridges among E299 (helix i), R309, and E312 seem to fasten the end of helix i. In the opposite side from this part, the end of helix a is clamped by salt bridges among E306, K317, and E30 (helix a). In the bottom of helix j, salt bridges among H343 (helix k), E312, and R342 (helix k) seem to fasten helix k. However, the bridges in HD711, HD708, and HD177 are broken because of mutations in the C-terminal region (13). The successions of ionic residues of E306, K310, D313, K317, and E321 cover the exterior of helix $\mathrm{j}$ facing to the solvent. Like residues in the exterior of helix e, these charged residues stabilize helix $\mathrm{j}$ with the electrostatic networks in each turn of helix. The interior of helix $\mathrm{j}$ consists of hydrophobic residues of L307, A308, L310, V311, A314, V315, A 316, A318, L319, and L320. These residues produce a hydrophobic pocket among helices $\mathrm{j}, \mathrm{i}, \mathrm{a}$, and $\mathrm{k}$ therefore this results an inelastic conformation in the C-terminal region of IPMDH. Residue of V305 at the top helix $\mathrm{j}$, accompanied by a hydrophobic loop i-j, may control the width-1 of the minor groove (Figure 4). In addition, residues of L319 and L320 in the bottom region of helix $\mathrm{j}$ form a hydrophobic pocket with residues of L112 (loop F-G), L256 (loop h-E), and L327 ( $3_{10}$ helix, between helices $\mathrm{j}$ and $\mathrm{k}$ ). This pocket seems to regulate the width- 2 of minor groove. To open the minor groove theoretically, entire helix $\mathrm{j}$ should be pushed toward strand D to close the active site groove in the opposite site. It can be achieved presumably by enlarging both ends of the width of minor groove simultaneously.

The width- 1 of minor groove in the top of helix $\mathrm{j}$ is measured from the distances between $\mathrm{C} \alpha$-atoms of K175 and V305 and the width- 2 in the bottom from the distance between $\mathrm{C} \alpha$-atoms A316 and F109 (Figure 4). The former has been reported (13). The average values in the later are 6.40, 7.17, 6.16, and $6.15 \AA$, for A172L, HD711, HD708, and HD177, respectively.

First Domain Movement The electrostatic and hydrophobic effects have been suggested as contributing factors to protein stability. These, however, are not be able to explain the phenomena in HD708 and HD177 which have larger hydrophobic interactions than that of HD711 but less thermostable (13). The motion of the domain in a particular circumstance may elucidate the stability. The motion of IPMDH in solution have been detected from small-angle X-ray scattering experiments, in which the active site of the enzyme was in closed states upon binding NADH and IPM (20). The localized motions of the protein in crystal are also observed in a temperature-jump time-resolved X-ray crystallographic technique (21). The motions of IPMDH domains are found from their crystal structures. 


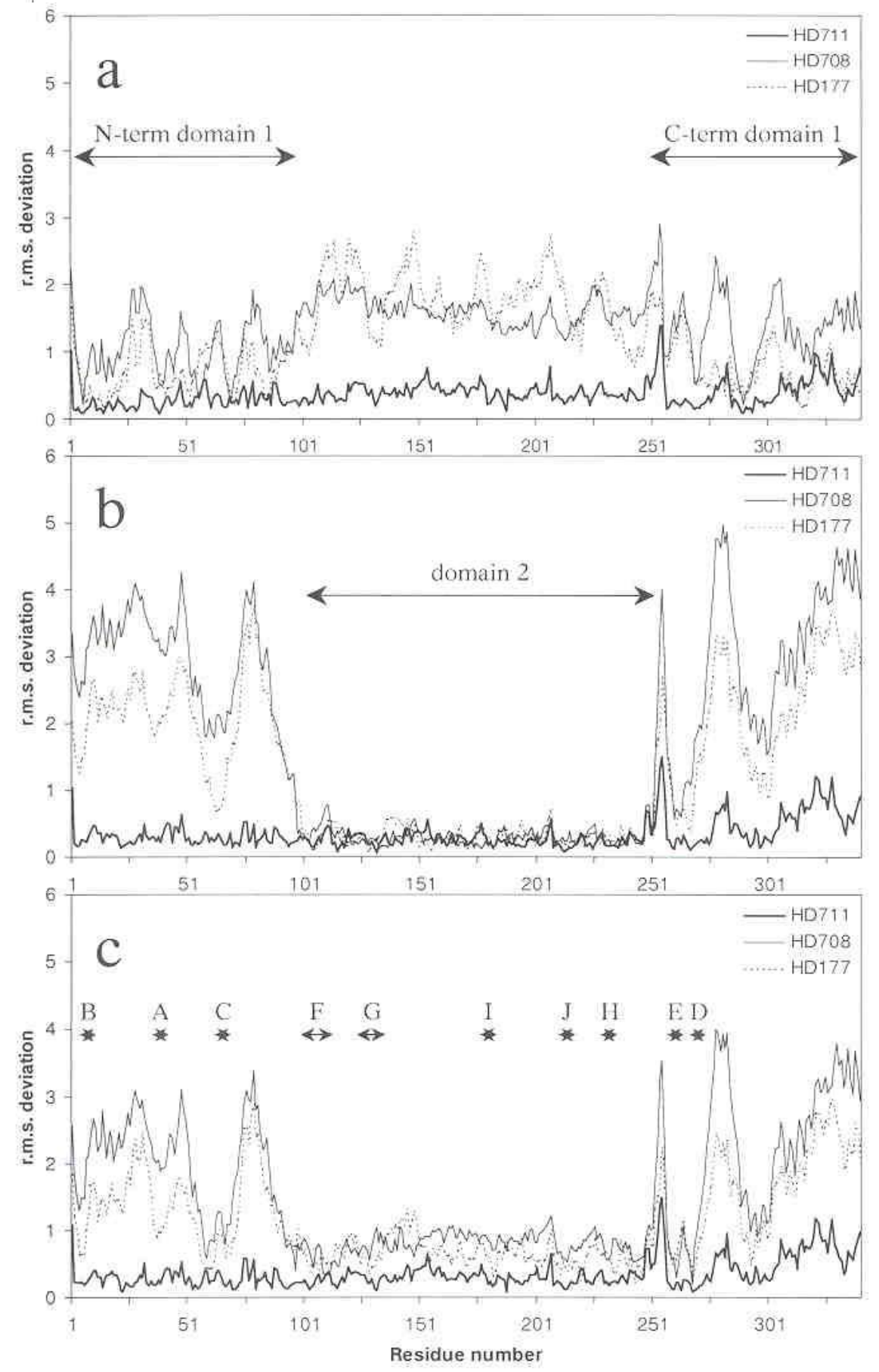




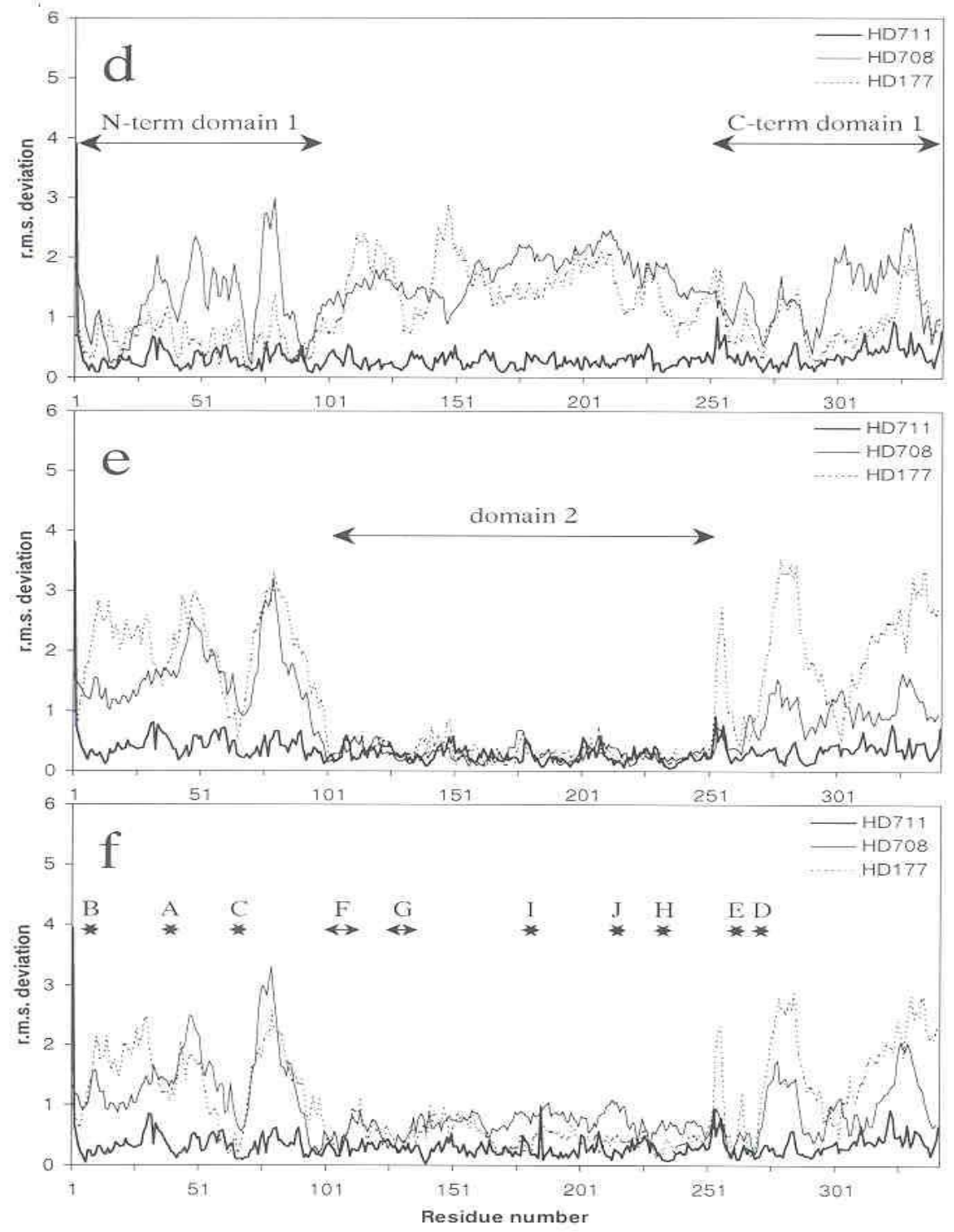

Figure 5 Root mean square deviations (in $\AA$ ) of backbone atoms showing the discrepancies in the conformation relative to $\mathrm{A} 172 \mathrm{~L}$ of the three molecules IPMDHs HD711, HD708, and HD177. The molecules are superimposed onto the backbones domain 1 (residues 1-99, N-term and 252-340, C-term) (a and d), domain 2 (residues 100-251) (b and e), and pillar (residues 2-7, B, 35-40, A, 66-69, C, 100-110, F, 126-133, G, 179-184, I, 210-215, J, 232-236, H, 258263, E and 267-270, D) (c and f). The plots are for subunits A (a, b, and c) and B (d, e, and f). 
To analyze the motion of the domain, each of the three independent mutantIPMDH molecules was superimposed onto the A172L structure based on the domain 1, domain 2, and pillar and the root mean square (r.m.s.) deviations between two coordinate sets were calculated (Figure 5). HD711 structure has small displacements indicating similarity with A172L over the whole molecule (Figure 5a, 5b, 5d, and 5e). In contrast, HD708 and HD177 structures deviate from those of $\mathrm{A} 172 \mathrm{~L}$ and their plots show a domain movement (Figure 5a, 5b, $5 \mathrm{~d}$, and $5 \mathrm{e}$ ). As a simple way to analyze for domain motion in IPMDH structures, the r.m.s. deviations were calculated with the superposition based on pillar (Figure 5c and 5f). These figures show that the domain 1 in HD708 or HD177 moved larger than the domain 2. Like a paperclip motion, the domain 1 of IPMDH move to open-close the active site or minor groove through the pillar. The motion of the domain 1 shifts the strands A, B, C, D, and E (parts of the pillar in the first domain).

Compared with A172L pillar, the pillars of mutants HD711, HD708, and HD177 are distorted with overall r.m.s. deviations of 1.0, 1.7, and $1.8 \AA$, respectively. These conformational changes of pillars owing to mutations in the C-terminal region impel strand C closer to strand D (Figure 3). Strand D, therewith, push strand $\mathrm{E}$ away to the direction of minor groove. These motions bring a narrow minor groove. Hence the mutations in IPMDHs of HD711, HD708, and HD177 support that the closing-minor-groove motion results in the reduction of their thermostability.

\begin{tabular}{lccc}
\hline Type of energy & HD711 & HD708 & HD177 \\
\hline Bond & 5 & 96 & 5 \\
Angle & -149 & -97 & -172 \\
Dihedral & 1429 & 1479 & 1695 \\
Improper & 263 & 186 & 2 \\
Lennard-Jones & 2444 & 2360 & 2738 \\
Electrostatic & 7 & 7 & 8 \\
Constraints, other & 0 & 0 & 0 \\
\hline Total & 3999 & 4031 & 4276 \\
\hline
\end{tabular}

${ }^{a}$ This value is calculated with the dielectric constant $\left(\varepsilon_{0}\right)$ value of 80 .

Table 2 The change of potential energy (in kcal per mol) of mutant IPMDHs.

To detect the conformational change, the discrepancies of potential energy relative to A172L of each mutant IPMDHs are calculated with the program CHARMM. The energy based on the first 340-residues of dimer IPMDH is shown in Table 2. This table shows that the motion in IPMDH mutant strain dihedral angles and produce close-contacts. These phenomena were identified with significant changes in the dihedral and Lennard-Jones energies. The increase of the total of potential energy of IPMDH molecules correlates with 
their thermal instability. These results are consistent with the result of thermostability measurement that the $\mathrm{T}^{1 / 2}$ (temperature for $50 \%$ remaining activity) of IPMDHs reduce in an order of A172L $>$ HD711 > HD708 > HD177 (15).

The motion of the first domain of IPMDH changes wide grooves. Strand D consisting of residues P267, V268, F269 and E270 seem to be a hinge-bending region of the movement. The accessible surface areas of these residues, calculated with radius probe of $1.4 \AA$ and a $\mathrm{Z}$ spacing factor of 0.1 , show that all residues are buried inside the molecule. The solvent can introduce no atoms V268 and F269 in all mutants. Residues of P267 and E270 in all mutants have a fractional accessibility of the whole residue/side chain of about 0.20 and 0.15 , respectively.

Two strands (C and E) and two helices ( $\mathrm{i}$ and $\mathrm{d}$ ) coated strand $\mathrm{D}$ in the first domain of IPMDH. The side chains of P267 and F269 face to helix i and side chains of V268 and E270 to helix d. The large side chain of F269 fills up the hydrophobic core of A66 (strand C), A260 (strand E), P267, and M297 (helix i). Residue of V268 forms hydrophobic interactions with residues of V67 (strand C), L93 (helix d), and L99 (loop d-F). Although residue of E270 is buried in hydrophobic residues of L69 (strand C) and L90 (helix d), it makes a salt bridge with R94 (helix d). The folding topology of IPMDH insulates strand D inside the molecule but the strand have potential to move toward helices i or d (Figure $3)$.

All atoms in strand D of each mutant IPMDH are observed in the electron density map obviously (data not shown). Furthermore, the average temperature factors for backbone atoms and side chains (in the parentheses) in strand D of A172L, HD711, HD708, and HD177 are 18 (21), 20 (21), 35 (37), and $21 \AA^{2}$ $\left(20 \AA^{2}\right)$, respectively. These results suggest that no significant thermal motion changes on strand D except for HD708 (slightly higher). The detailed hydrogen bonds between the strand $\mathrm{D}$ and adjacent strand (C or E) among mutants are also conserved. These results reveal that distorted strand D is not caused by disordered structure but reasonably by a movement of the first domain.

The facts that the strand $\mathrm{D}$ (hinge-bending region) is parallel to helix $\mathrm{j}$ suggest an idea how to engineer IPMDH. The proposed model to enhance thermostability of IPMDH suggests that the motion of first domain to enlarge minor groove can be accomplished through the pushed helix $\mathrm{j}$ away (Figure 6). The consecutive motions of helix $j$, helix $i$, strand $D$, and helix $d$ in the first domain of the enzyme seem to close the active site groove on the opposite side (arrows in Figure 6). At the same time, the motion hopefully effects movements of strand $\mathrm{E}$ and loop i-D away from each other. It is predictable from these 
motions that the substrate-binding and coenzyme-binding clefts in the active site groove will be closer and farther, respectively. The closed conformation of the active site groove gives high barrier conformational protein for substrate binding (22). It means the induction of the enzyme activity needs a high energy and the enzyme becomes more thermostable.

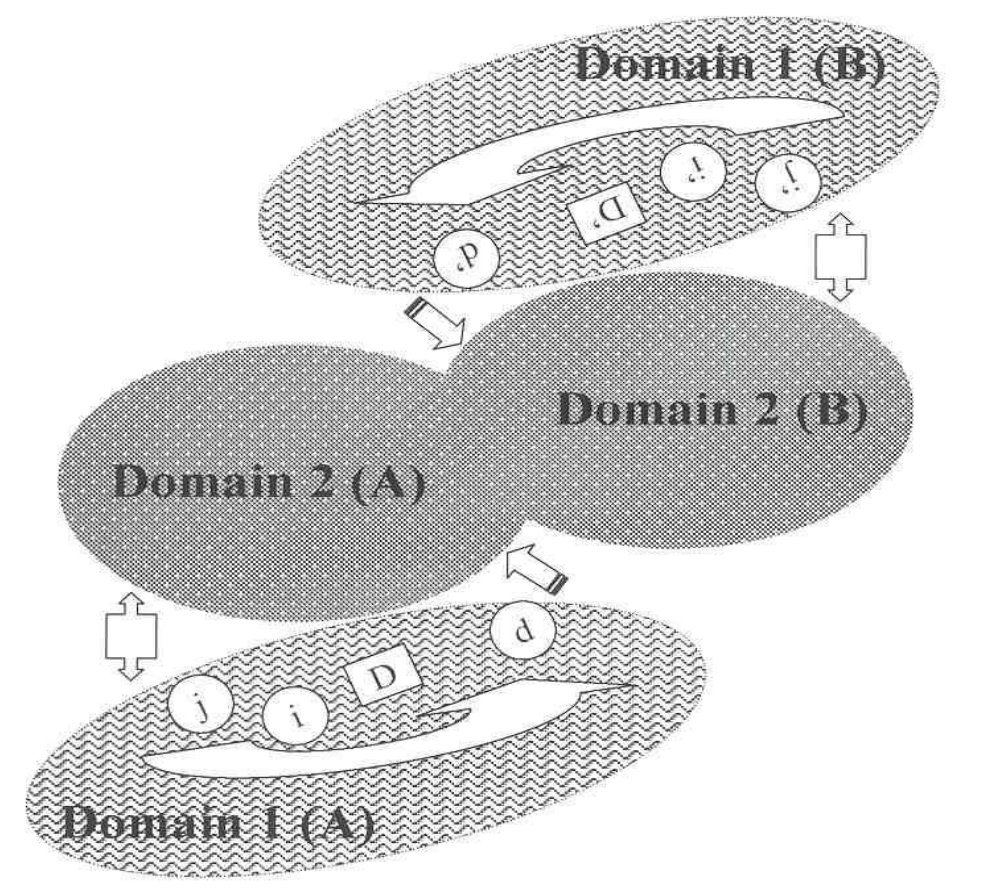

Figure 6 Proposed mechanism model to enhance thermal stability of IPMDH. Circles (lower-case letters) and boxes (upper-case letters) represent $\alpha$-helices and $\beta$-strands, respectively.

\section{$4 \quad$ Summary}

T. thermophilus IPMDH is more thermostable when the active site groove is closed. However, IPMDH is also an induced-fit enzyme, where the active site groove is closed upon binding the substrate (20). The structural analyses of IPMDHs describes here have identified a particular motion to enhance thermal stability. Site directed mutagenesis at chosen positions could be used to examine this model. Consider both width of minor groove shown in Figure 4. Changing V305 with I, L, or F would increase repulsion to K175 and replacing A316 to V, I, or L may not change a hydrophobic interaction with F109 except 
expand the distance. These double mutations will stretch the minor groove out and the motion of domain may close the active site. Some efforts to make variant IPMDHs have already been performed and their crystal structures have been solved. IPMDH structures, in which A172 replaced with G, V, I, or F, reveal the correlation of the expanded minor groove to the thermal stability. Although the increase of the thermostability is not satisfied yet, the proposed strategy from this study is worth trying.

\section{Acknowledgement}

Z. N. thanks the Department of Chemistry, Bandung Institute of Technology for providing a doctoral fellowship in part by the Center Grant Project and by the OECF Project.

\section{References}

1. Wallon, G., Lovett, S. T., Magyar, C., Svingor, A., Szilagyi, A., Zàvodsky, P., Ringe, D. \& Petsko, G. A., Sequence and homology model of 3-isopropylmalate dehydrogenase from the psychotropic bacterium Vibrio sp. I5 suggest reasons for thermal instability, Prot. Eng. 10, 665672 (1997).

2. Sekiguchi, T., Suda, M., Ishii, T., Nosoh, Y. \& Tsuda, K., The nucleotide sequence of 3-isopropylmalate dehydrogenase gene from Bacillus caldotenax, Nucl. Acids Res. 15, 853 (1987).

3. Sekiguchi, T., Ortega-Cesena, J., Nosoh, Y., Ohashi, S., Tsuda, K. \& Kanaya, S., DNA and amino acid sequences of 3-isopropylmalate dehydrogenase of Bacillus coagulans. Comparison with the enzyme of Saccaromyces cerevisiae and Thermus thermophilus, Biochim. Biophys. Acta 867, 36-44 (1986).

4. Andreadis, A. \& Rosenthal, E. R., The nucleotide sequence of leuB from Salmonella typhimurium, Biochim. Biophys. Acta 1129, 228-230 (1992).

5. Imai, R., Sekiguchi, T. Nosoh, Y.\& Tuda, K., The nucleotide sequence of 3-isopropylmalate dehydrogenase gene from Bacillus subtilis, Nucl. Acids Res. 15, 4988 (1987).

6. Kawaguchi, H., Inagaki, H., Kuwata, Y., Tanaka, H. \& Tano, T., 3isopropylmalate dehydrogenase from chemolithoautotroph Thiobacillus ferrooxidans: DNA sequence, enzyme purification, and characterization, J. Biochem. 114, 370-377 (1993).

7. Kagawa, Y., Nojima, H., Nukiwa, N., Ishizuka, M., Nakajima, T., Yasuhara, T., Tanaka, T. \& Oshima, T., High guanine plus cytosine content in the third letter of codons of an extreme thermophile. DNA 
sequence of the isopropylmalate dehydrogenase of Thermus thermophilus, J. Biol. Chem. 259, 2956-2960 (1984).

8. Kirino, H. \& Oshima, T., Molecular cloning and nucleotide sequence of 3-isopropylmalate dehydrogenase gene (leuB) from an extreme thermophile, Thermus aquaticus YT-1. J. Biochem. 109, 852-857 (1991).

9. Imada, K., Sato, M., Tanaka, N., Katsube, K., Matsuura, Y. \& Oshima, T., Three-dimensional structure of highly thermostable enzyme, 3isopropylmalate dehydrogenase of Thermus thermophilus at $2.2 A$ resolution. J. Mol. Biol. 222, 735-738 (1991).

10. Magyar, C., Szilágyi, A. \& Závodszky, P., Relationship between thermal stability and 3-D structure in a homology model of 3-isopropylmalate dehydrogenase from Escherichia coli, Prot. Eng. 9, 663-670 (1996).

11. Wallon, G., Kryger, G., Lovett, S. T., Oshima, T., Ringe, D. \& Pestko, G. A., Crystal structures of Escherichia coli and Salmonella typhimurium 3isopropylmalate dehydrogenase and comparison with their thermophilic counterpart from Thermus thermophilus, J. Mol. Biol. 266, 1016-1031 (1997).

12. Rhode, D. J. \& Martin, B. L., Localized structural effects of electrostatic interactions in a thermostable Enzyme, Biochem. Biophys. Res. Commun. 258, 178-183 (1999).

13. Nurachman, Z., Akanuma, S., Sato, T., Oshima, T. \& Tanaka, N., Crystal structures of 3-isopropylmalate dehydrogenases with mutations at the $C$ terminus: analyses of structure-stability relationships, Prot. Eng. 13, 253-258 (2000).

14. Qu, C., Akanuma, S., Moriyama, H., Tanaka, N. \& Oshima, T., A mutation at the interface between domains causes rearrangement of domains in 3-isopropylmalate dehydrogenase, Prot. Eng. 10, 45-52 (1997).

15. Akanuma, S., Yamagishi, A., Tanaka, N. \& Oshima, T., Spontaneous tandem sequence duplications reverse the thermal stability of carboxylterminal modified 3-isopropylmalate dehydrogenase, J. Bacteriol. 178, 6300-6304 (1996).

16. Jones, T. A., Interactive computer graphics: FRODO, Methods Enzymol. 115, 157-171 (1985).

17. Brünger, A. T., A System for X-ray Crystallography and NMR, X-PLOR, Version 3.1, Yale University Press, New Heaven (1992).

18. Kraulis, P. J., MOLSCRIPT: a program to produce both detailed and schematic plots of protein structures, J. Appl. Cryst. 24, 946-950 (1991).

19. Akanuma, S., Qu, C., Yamagishi, A., Tanaka, N. \& Oshima, T., Effect polar side chains at position 172 on thermal stability of 3isopropylmalate dehydrogenase from Thermus thermophilus, F.E.B.S. Lett. 410, 141-144 (1977). 
20. Kadono, S., Sakurai, M., Moriyama, H., Sato, M., Hayashi, Y., Oshima, T. \& Tanaka, N., Ligand-induced changes in the conformation of 3isopropylmalate dehydrogenase from Thermus thermophilus, J. Biochem. 118, 745-752 (1995).

21. Hori, T., Moriyama, H., Kawaguchi, J., Hayashi-Iwasaki, Y., Oshima, T. \& Tanaka, N., The initial step of the thermal unfolding of 3isopropylmalate dehydrogenase detected by the temperature-jump Laue method, Prot. Eng. 13, 527-533 (2000).

22. Ma, B., Kumar, S., Tsai, C-J. \& Nussinov, R., Folding funnels and binding mechanisms, Prot. Eng. 12, 713-720 (1999). 